\title{
Abundance of Flt3 and its ligand in astrocytic tumors
}

This article was published in the following Dove Press journal:

OncoTargets and Therapy

23 May 2013

Number of times this article has been viewed

\author{
C Eßbach' \\ N Andrae' \\ D Pachow' \\ J-P Warnke ${ }^{2}$ \\ A Wilisch-Neumann' \\ E Kirches' \\ C Mawrin' \\ 'Department of Neuropathology, \\ Otto-von-Guericke University, \\ Magdeburg, ${ }^{2}$ Department \\ of Neurosurgery, Paracelsus Hospital, \\ Zwickau, Germany
}

Correspondence: Elmar Kirches Department of Neuropathology, Otto-von-Guericke University, Leipziger Strasse 44, 39120 Magdeburg, Germany

Tel +4939 I67I 58I3

Fax+4939 1671 3300

Email elmar.kirches@med.ovgu.de
Background: Molecular targeted therapies for astrocytic tumors are the subject of growing research interest, due to the limited response of these tumors, especially glioblastoma multiforme, to conventional chemotherapeutic regimens. Several of these approaches exploit the inhibition of receptor tyrosine kinases. To date, it has not been elucidated if fms-like tyrosine kinase- 3 (Flt3) and its natural ligand (Flt3L) are expressed in astrocytic tumors, although some of the clinically intended small-molecule receptor tyrosine kinase inhibitors affect Flt3, while others do not. More importantly, the recent proof of principle for successful stimulation of the immune system against gliomas in preclinical models via local Flt3L application requires elucidation of this receptor tyrosine kinase pathway in these tumors in more detail. This therapy is based on recruitment of Flt3-positive dendritic cells, but may be corroborated by activity of this signaling pathway in glioma cells.

Methods: Receptor and ligand expression was analyzed by real-time polymerase chain reaction in 31 astrocytic tumors (six diffuse and 11 anaplastic astrocytomas, 14 glioblastomas) derived from patients of both genders and in glioblastoma cell lines. The two most common activating mutations of the Flt3 gene, ie, internal tandem duplication and D835 point mutation, were assessed by specific polymerase chain reaction.

Results: A relatively high abundance of Flt3L mRNA ( $4 \%-6 \%$ of the reference, $\beta_{2}$ microglobulin) could be demonstrated in all tumor samples. Flt3 expression could generally be demonstrated by 40 specific polymerase chain reaction cycles and gel electrophoresis in $87 \%$ of the tumors, including all grades, although the small quantities of the receptor did not allow reliable quantification. Expression of both mRNAs was verified in the cell lines, excluding a derivation solely from contaminating lymphocytes or macrophages. No activating mutations were found.

Conclusion: Our results warrant further analysis of endogenous Flt3 signaling in these tumors prior to application of immunotherapy in human patients.

Keywords: fms-like tyrosine kinase-3, fms-like tyrosine kinase-3 ligand, glioblastoma multiforme, receptor tyrosine kinases

\section{Introduction}

A variety of receptor tyrosine kinases (RTKs) are already a focus of interest in glioma research, because they often show mutations promoting tumor development. ${ }^{1,2}$ Treatments attempting to inhibit either the kinase activity or downstream elements of its signaling pathway are common principles of modern "targeted" therapeutic approaches. ${ }^{1,3}$ Activating mutations, gene amplification, and enhanced expression of various RTKs have been found in brain tumors, including glioblastoma multiforme (GBM), ${ }^{2}$ making RTK-based treatment interesting in these tumors, which show pronounced resistance to conventional chemotherapeutic drugs. 
The alkylating agent temozolomide, used in addition to surgery and radiotherapy, is able to prolong the survival of patients with GBM. ${ }^{4}$ The prognosis remains poor, and depends strongly on promoter methylation of $\mathrm{O}^{6}$-methylguanine-DNA methyltransferase, which represents a major mechanism of chemoresistance against alkylating drugs. ${ }^{2,4}$

Despite the progress made by introduction of temozolomide as the gold standard of chemotherapy for GBM, the poor prognosis continues to drive an intensive search for targeted therapies. For two of these approaches, RTKs play important but opposite roles. The more widely followed strategy involves using RTKs in the plasma membranes of tumor cells or endothelial cells of tumor blood vessels as a potential pharmacologic target to attack the tumors or their vasculature directly by inhibitors, which usually block signaling of a variety of RTKs in parallel..$^{5-8}$ On the other hand, the ligand of fms-like tyrosine kinase-3 (Flt3) has been used for successful stimulation of the immune system against glioma xenografts in animal models. ${ }^{9-12}$ This suggests that local immune stimulation against glioma cells may be suitable for creating a new gene therapy, eventually if combined with a cytotoxic gene therapy, eg, ganciclovir, after adenoviral transfer of thymidine kinase to the tumor. ${ }^{10-12}$ For both approaches, it is essential to know if wild-type or mutant Flt3 is expressed in human gliomas.

Although Flt3 signaling in gliomas has not been well investigated as yet, a growing body of molecular evidence connects other RTKs to astrocytic tumors. Primary GBM, developing rapidly without any evidence for less malignant precursor lesions, constitutes $90 \%-95 \%$ of cases and represents the most malignant glial tumor. The EGFR/PTEN/ Akt/mTor pathway is a well established molecular route leading to enhanced or even constitutive transmission of epidermal growth factor receptor signals in primary GBM. This can happen by alteration of various components of the signaling cascade, ${ }^{3}$ eg, overexpression or amplification of epidermal growth factor receptor, ${ }^{13}$ which may be associated with activating gene mutations,,${ }^{14}$ or by loss or inactivation of the tumor suppressor, PTEN, which antagonizes this pathway. ${ }^{2}$ By ligand binding and dimerization, the epidermal growth factor receptor recruits phosphoinositide-3 kinase, which causes formation of PIP3, leading to activation of downstream kinases like Akt and mTor. The results of Akt activation are cell proliferation and blockade of apoptosis, which are important factors in carcinogenesis. The downstream element, mTOR, regulates biosynthesis of protein, which is a prerequisite for cell growth prior to cell division. ${ }^{1,3,15}$ The phosphatase PTEN antagonizes Akt by dephosphorylation of the lipid second messenger, PIP3, thereby restricting cell growth and proliferation. Functional loss of this antagonistic element, ie, PTEN, by mutation or promoter methylation is a frequent event in primary GBM. Similar signaling cascades are also used by other RTKs in gliomas, eg, the platelet-derived growth factor receptor. Today, inhibition of these pathways, either at the receptor level or by targeting downstream elements (eg, mTOR), is recognized as an opportunity for modern treatment. ${ }^{1-3,15-17}$

Because it is currently unknown if Flt3 is expressed in gliomas, it cannot be stated that efficient RTK inhibitors for glioma therapy must be active against this target and eventually its mutant variants. The intended use of the Flt3 ligand (Flt3L) is to attract and proliferate antigen-presenting dendritic cells within the GBM tumor as part of an improved gene therapy approach. ${ }^{9,11,12}$ It will be important to know if expression of the Flt3 receptor may corroborate the whole approach, and whether or not it is restricted to blood cell precursors. Flt3 in tumor cells may enhance tumor growth using such immunotherapy.

Flt3, a class III RTK (also known as human stem cell tyrosine kinase-1 or fetal liver kinase-2) is known to have a crucial role in normal hematopoiesis. ${ }^{18} \mathrm{~A}$ variety of mutations of the Flt3 receptor are found in different hyperproliferative diseases of blood cell precursors, eg, acute myeloid leukemia and acute lymphoblastic leukemia. The most common mutations of Flt3 in acute myeloid leukemia cells are internal tandem duplications ${ }^{19}(20 \%-25 \%$ of cases $)$ and a D835 point mutation in the activation loop (7\% of cases) that leads to hyperproliferation and drug resistance. ${ }^{20}$ Although internal tandem duplications of variable length occur, they all arise in the same domain and constitutively activate the kinase.

The Flt3L came into focus as a research interest because its induced overexpression was used successfully as a novel treatment for GBM in preclinical models. ${ }^{9-12}$ Its ability to activate the immune system via antigen-presenting dendritic cells leads to an immune attack against the tumor, eliminating it in animal experiments. ${ }^{9}$ Meanwhile, it was demonstrated that this therapeutic success resulted from immunostimulation, given that it also occurred in xenografts on the contralateral side of the brain which had not been injected with the gene vectors. ${ }^{12}$ However, the Flt3 receptor has never been examined in GBM tissue or cell cultures. If expressed in tumor cells, a role in proliferation and apoptosis may be suggested, similar to those of other RTKs, which may counteract the abovementioned therapeutic effects of the ligand in human patients. In the 
present study, we analyzed the expression of this RTK and its ligand and screened the receptor gene for the most common activating mutations.

\section{Materials and methods Tissue samples and cell culture}

Frozen human tissue glioma samples were taken from the files of the department of neuropathology. Based on histologic examination, all samples were assigned by an experienced neuropathologist (CM) to one of the following tumor entities: diffuse astrocytoma (A, World Health Organization grade II), anaplastic astrocytoma (AA, World Health Organization grade III) and glioblastoma multiforme (GBM, World Health Organization grade IV).

Human glioblastoma cell lines (U87MG, U138MG, U251MG) were purchased from the American Type Culture Collection (Manassas, VA, USA), while LN229 and LN405 were sourced from the Deutsche Sammlung für Mikroorganismen und Zellkulturen (Braunschweig, Germany). The tumor cell lines were cultured at $37^{\circ} \mathrm{C}$ in high-glucose Dulbecco's Modified Eagle's medium (PAA, Linz, Austria) supplemented with $10 \%$ fetal calf serum and a mixture of penicillin and streptomycin. The identity of the cell lines was confirmed using the AmpFSTR kit and GeneMapper ID version 3.2 software from Applied Biosystems (Foster City, CA, USA), which automatically determines the alleles of polymorphic DNA markers on various chromosomes and the alleles of the sex marker, amelogenin.

\section{Real-time polymerase chain reaction}

A total of 31 tumors $(6 \times \mathrm{A}, 11 \times \mathrm{AA}, 14 \times \mathrm{GBM})$ derived from patients of both genders and the cell lines were examined. Total RNA was isolated with Trizol reagent (Life Technologies, Darmstadt, Germany), and $1 \mu \mathrm{g}$ of total RNA was reverse-transcribed into cDNA using hexanucleotide random primers from Bioline (Luckenwalde, Germany).

Quantitative real-time polymerase chain reaction (PCR) was performed using an ABI Prism 7000-SDS machine (Applied Biosystems) with $1 \mu \mathrm{L}$ of cDNA, ABI SYBR Green PCR Master Mix (Applied Biosystems), and the following primers at an annealing temperature of $55^{\circ} \mathrm{C}$ : AATGATGATGCCCAATTTACC (forward) and GTAACACTTCTTCCAGGTCC (reverse) for Flt3, AGACTGTCGCTGGGTCCAAG (forward) and GCTCGGAGGTCTCCTGCAG (reverse) for Flt3L, CCAGCAGAGAATGGAAAGTC (forward) and GATGCTGCTTACATGTCTCG (reverse) for $\beta 2$ microglobulin, which was used as a reference for normalization of $\mathrm{C}_{\mathrm{t}}$ values. Negative controls without a template were included in each experiment. All reactions were run in duplicate. The $\mathrm{C}_{\mathrm{t}}$ values were automatically calculated by the software in the PCR machine. Relative expression levels (E) of the target genes were expressed as:

$$
\mathrm{E}=1 / 2^{\Delta \mathrm{Ct}} \text {, with } \Delta_{\mathrm{Ct}}=\mathrm{C}_{\mathrm{t}}(\text { target })-\mathrm{C}_{\mathrm{t}}(\beta 2) \text {. }
$$

All melting curves were checked for the appropriate $\mathrm{T}_{\mathrm{m}}$ values. Due to the extremely high $\mathrm{C}_{\mathrm{t}}$ values (around 30), ie, low abundance, observed for the receptor mRNA, the reaction products of the Flt3 system were separated on $8 \%$ polyacrylamide gel by electrophoresis and silver-stained. This was done in order to visualize a single PCR product in addition to verifying PCR specificity by the $T_{m}$ values.

\section{Mutation analysis}

A total of 46 tumors $(6 \times \mathrm{A}, 6 \times \mathrm{AA}, 34 \times \mathrm{GBM})$ derived from patients of both genders were examined. Negative controls without a template were run with each experiment. Glioblastoma-derived cell lines were included for analysis of internal tandem duplications. Total genomic DNA was extracted from the cells and tissues using a DNA extraction kit from Macherey-Nagel (Düren, Germany) according to the manufacturer's instructions. For analysis of internal tandem repeats, a PCR was carried out with the primers GCAATTTAGGTATGAAAGCCAGC (forward) and CTTTCAGCATTTTGACGGCAACC (reverse), while the TCCAGGATAATACACATCACAG (forward) and ACCATGATAACGACACAACAC (reverse) primers were used for analysis of the point mutation, each at an annealing temperature of $55^{\circ} \mathrm{C}$.

The forward primers intended for analysis of internal tandem duplications were $5^{\prime}$-labeled with 6-FAM fluorescent dye in order to amplify DNA fragments, which were visible during capillary electrophoresis on a fluorescent capillary sequencer (ABI-310 Genetic Analyzer, Applied Biosystems). Following electrophoresis together with ROXlabeled standard fragments (ROX-standard 500, Applied Biosystems), the lengths of the 6-FAM-labeled fragments were automatically calculated by the GeneScan software (Applied Biosystems). The length of the amplicon was 330 bp for chromosomal DNA without internal tandem duplications. The precise value calculated by GeneScan according to the retention time was $330.07 \pm 0.37 \mathrm{bp}$ (mean \pm standard deviation). While GeneScan results were reproducible within a range of one bp between minimal and maximal values obtained in the same capillary run, the expected mean fragment length in the event of internal 
tandem duplications was around $400 \mathrm{bp}$, and fragments up to $500 \mathrm{bp}$ were analyzed.

The PCR products were analyzed using a PCR restriction fragment length polymorphism method for detection of the D835 point mutation. The amplicon has a length of $237 \mathrm{bp}$. The amplified DNA was cut using the EcoRV enzyme (New England Biolabs, Beverly, MA, USA) according to the manufacturer's instructions. The enzyme creates two fragments (97 bp and $140 \mathrm{bp}$ ) of wild-type DNA. Mutant DNA is not cut because of deletion of the recognition site for EcoRV, which includes the mutated codon. The reaction products were separated on $9 \%$ polyacrylamide gel by electrophoresis and silver-stained.

\section{Statistical analysis}

All statistical analyses were performed using the Statistical Package for Social Sciences version 18 (SPSS Inc., Chicago, IL, USA). Statistically significant differences between the three groups were determined using analysis of variance and the Tukey post hoc test, and between two groups using the $t$-test. The significance level of all tests was set at $P \leq 0.05$.

\section{Results mRNA abundance in ligand and receptor by real-time PCR}

A total of 31 tumors $(6 \times \mathrm{A}, 11 \times \mathrm{AA}, 14 \times \mathrm{GBM})$ were examined. Expression of Flt3L mRNA was clearly demonstrated in all samples analyzed. $C_{t}$ values were sufficiently low to allow reproducible quantification. The abundance of Flt3L mRNA was in the range of $4 \%-6 \%$ of the abundance of the reference gene, $\beta 2$ microglobulin, in all human tumor samples (Figure 1A). To exclude the possibility that most of this Flt3L expression was the result of contamination of the mRNA pool by lymphocytes, macrophages, or other nontumor cells of tissue homogenates, the experiment included the glioblastomaderived cell lines, U87MG and U251MG, which showed $\beta 2$ microglobulin-normalized Flt3L expression of $8 \%$ and $25 \%$, respectively.

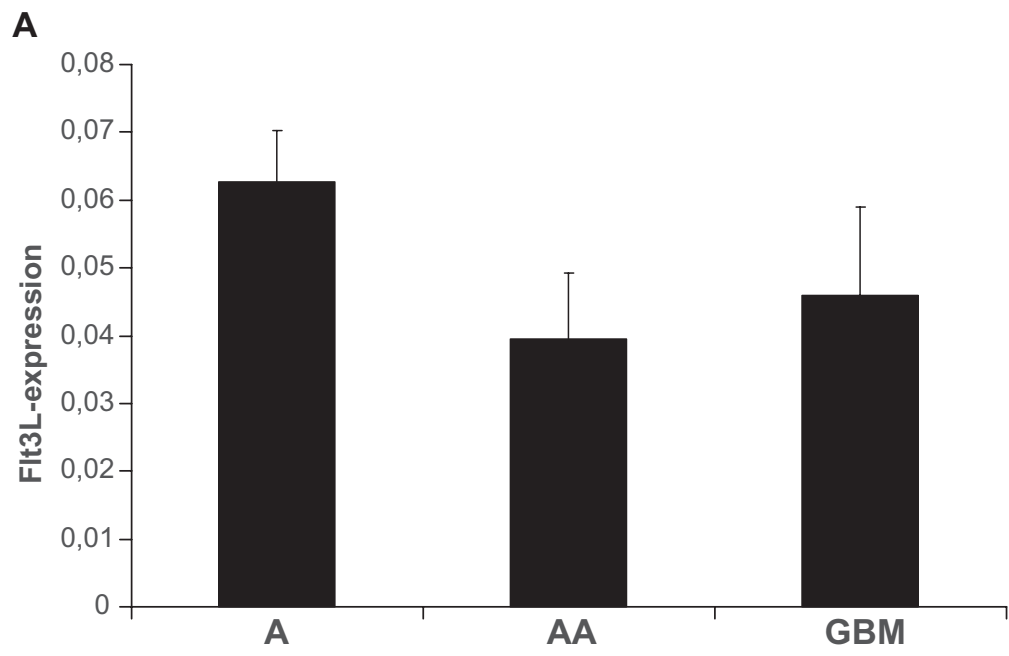

B

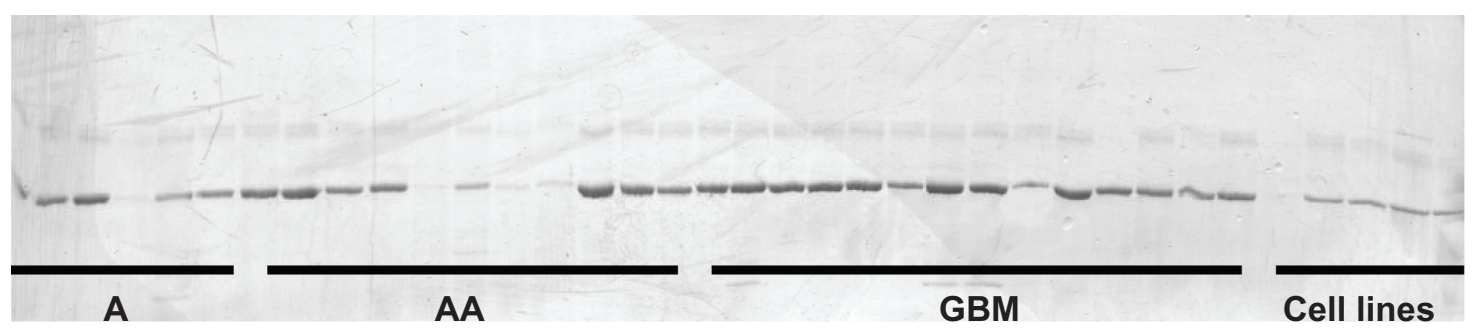

Figure I Expression of Flt3 receptor and ligand in astrocytic gliomas. (A) Real-time polymerase chain reaction quantification of Flt3L mRNA abundance in three diffuse astrocytomas of WHO grade II (A), six anaplastic astrocytomas of WHO grade III (AA) and I4 glioblastomas (GBM) of sufficient RNA quality for analysis. Means and standard errors of the mean are shown. (B) Silver-stained polyacrylamide gel, which identifies expression of Flt3 receptor mRNA in most diffuse astrocytomas of WHO grade II (A), most anaplastic astrocytomas of WHO grade III (AA), and glioblastomas (GBM), as well as all the glioblastoma-derived cell lines with the exception of U25IMG (outermost left from the lanes representing cell lines).

Notes: All available samples were used for this assay. Faint or missing bands in the human tumor samples corresponded to low RNA quality, ie, high $\mathrm{C}_{\mathrm{t}}$ values for the $\beta 2$ microglobulin housekeeping gene.

Abbreviations: WHO, World Health Organization; GBM, glioblastoma multiforme; Flt3, fms-like tyrosine kinase-3; Flt3L, fms-like tyrosine kinase-3 ligand. 
These results indicate that the ligand is among the genes of intermediate expression in human astrocytic tumors of all World Health Organization grades, occurring in adult patients (grades II-IV). Based on the low number of diffuse astrocytomas (grade II) available with sufficient RNA quality to be analyzed in this assay $(n=3)$, a statistically significant difference could not be detected between any of the three groups, nor between diffuse astrocytomas on the one hand and all high-grade tumors on the other. At least it can be stated that the results depicted in Figure 1A do not suggest a gradual decrease in mRNA with increasing tumor grade, which in addition was not supported by the higher levels of Flt3L mRNA observed in the glioblastoma-derived cell lines.

Intermediate expression of ligand in astrocytic tumors would have a functional impact only in the presence of its corresponding receptor, ie, Flt3. However, the abundance of the receptor mRNA turned out to be much lower, making even reliable quantification by SYBR green-based real-time PCR impossible. This was due to the fact that the $\mathrm{C}_{\mathrm{t}}$ values were often in a range around 30 , which does not allow a reliable quantitative determination. To gain at least qualitative proof that the receptor was indeed expressed in human astrocytic tumors of all grades, the resulting PCR fragments were separated on $8 \%$ polyacrylamide gel and silver-stained. These fragments represent the reaction products of real-time PCR, which had been run for 40 cycles. It was recognized that the PCR indeed had amplified a single fragment of predicted size in five of six diffuse astrocytomas, in eight of 11 anaplastic astrocytomas, and in all 14 glioblastomas (Figure 1B). For this PCR, it was even more important to include controls from pure glioma cell cultures, because trace amounts of receptor mRNA may indeed be attributed to contaminating lymphocytes or macrophages. A qualitative demonstration of Tfl3 mRNA expression after 40 PCR cycles could be obtained from the five glioblastoma-derived cell lines, with the only exception being U251MG. Together, these results indicate ubiquitous expression of the receptor mRNA in all human astrocytic tumor entities of adulthood, but only in trace amounts.

\section{Analysis of internal tandem repeat duplications}

Because genomic DNA-based mutation analysis did not necessarily require human tumor samples, which had been snapfrozen in liquid nitrogen under optimal conditions, ie, with minimal time delay after surgery, the group of glioblastoma samples available for mutation screening was larger. A total of 46 tumors $(6 \times \mathrm{A}, 6 \times \mathrm{AA}, 34 \times \mathrm{GBM})$ and the five glioblastoma-derived cell lines were examined. According to automated analysis by the GeneScan software, all of the fragments analyzed for internal tandem duplications had a mean fragment length of $330.07 \pm 0.37 \mathrm{bp}$, which means that no evidence of an internal tandem duplication with an expected mean fragment length around $400 \mathrm{bp}$ could be found, irrespective of tumor grading. Figure 2A gives an example of each tumor grade. The same negative result was observed for all five glioblastoma cell lines.

\section{Analysis of $\mathrm{D} 835$ point mutation}

Screening for this mutation in the same tumor DNA samples was done using a PCR restriction fragment length polymorphism method. The amplicon length was $237 \mathrm{bp}$. The EcoRV enzyme creates two fragments ( $97 \mathrm{bp}$ and $140 \mathrm{bp}$ ) in wild-type DNA, while any mutation of codon 835 leads to deletion of the restriction site, resulting in a fragment size of $237 \mathrm{bp}$. The somatic D835 mutation in the tumor cells or any other somatic base substitution or deletion would be expected to yield similar amounts of digested and undigested DNA, resulting in three bands of the predicted sizes and of similar intensity in silver-stained polyacrylamide gels. Given that all 46 tumor samples showed only the two short fragments, no point mutation was evident, irrespective of tumor grade. Figure 2B shows some examples.

\section{Discussion}

Bearing in mind the intended use of RTK inhibitors in GBM therapy, ${ }^{6-8}$ and, most of all, the goal of selective local Flt3 receptor stimulation in GBM immunotherapy, ${ }^{9-12}$ it will be necessary to gain detailed information regarding expression and activity of the Ftl3/Flt3L system in murine and human gliomas.

For implementation of small molecule RTK inhibitors in clinical studies of GBM, 4,7 it may theoretically play a role if these molecules are able to block Flt3 kinase activity, eg, sunitinib, or not, eg, gefitinib. However, there is no evidence so far of significant participation of Flt3 signaling in glioma growth, but this would have required intentional focus on this issue. On the other hand, the hopeful developments in gene therapeutic approaches to enhance the number of differentiated dendritic cells by local Flt3L delivery ${ }^{9-12}$ require the absence of proproliferative or antiapoptotic responsiveness of glioma cells towards the ligand. Otherwise, immune therapy may be corroborated by adverse effects, which accelerate tumor growth. Even if Flt3-signaling is not the dominant RTK pathway in untreated glioma cells, administration of large 
A
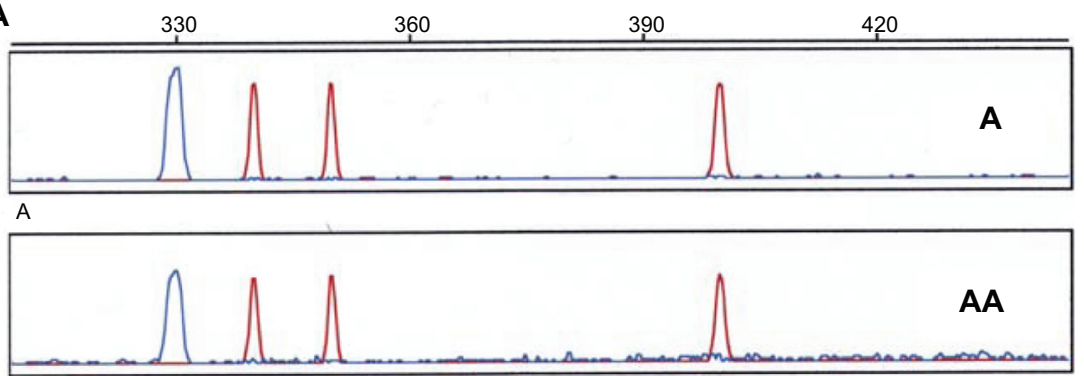

AA

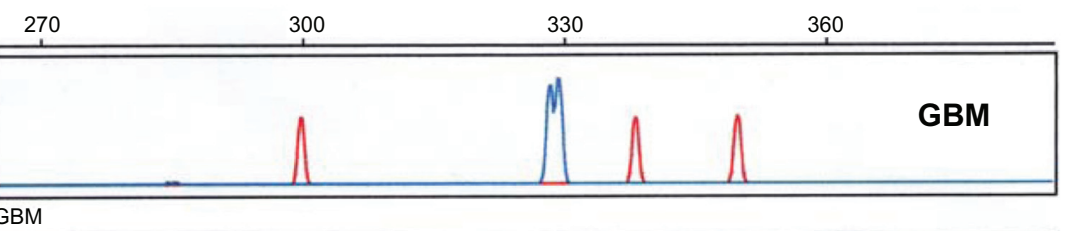

B

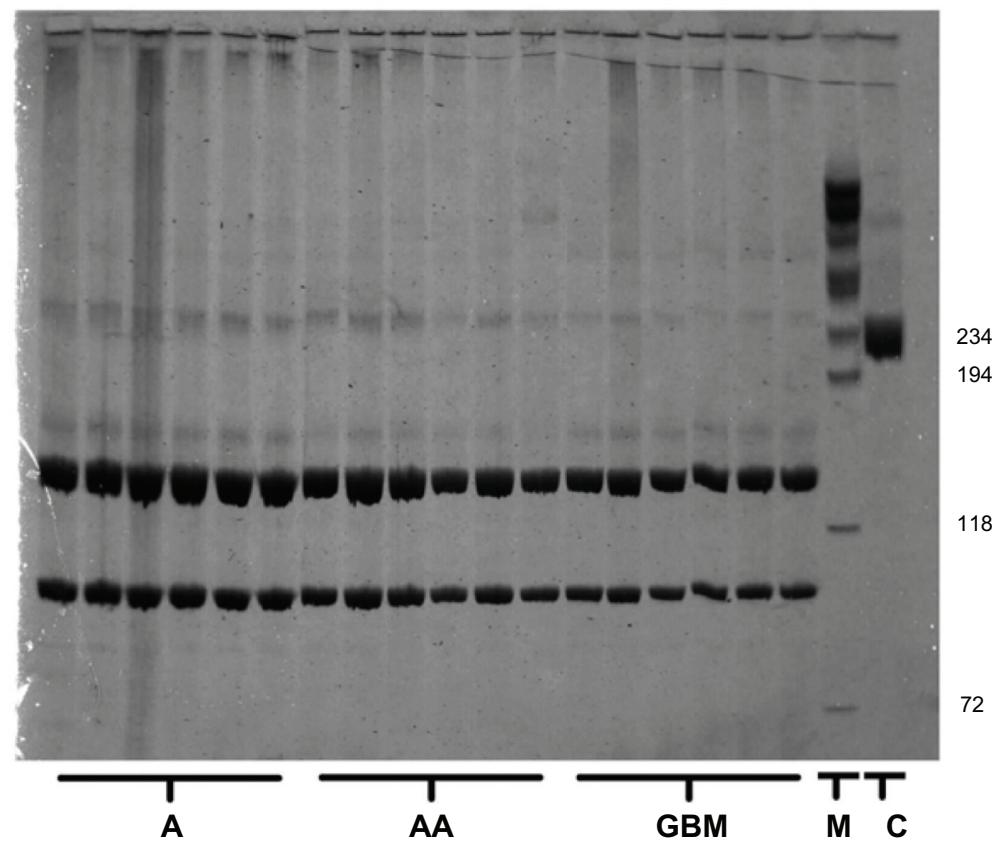

Figure 2 Absence of activating Flt3 mutations in astrocytic gliomas. (A) Examples of GeneScan analyses of 6-FAM-labeled polymerase chain reaction fragments spanning the Flt3 region, in which internal tandem duplications can occur. The tumor samples analyzed show the wild-type peak at a mean fragment length of $330.07 \pm 0.37$ bp. No sample contained additional peaks around 400 bp or of any size compatible with internal tandem duplications up to a size of 500 bp. The double peak in the GBM sample merely reflects an overlap between two peaks with a distance of 0.85 bp, which can be interpreted as a polymerase chain reaction artifact. (B) Diffuse astrocytomas (A), anaplastic astrocytomas (AA), and glioblastomas (GBM) were screened for the D835 point mutation.

Notes: An undigested product of the polymerase chain reaction (237 bp) served as a control (C) to indicate the expected fragment size and intensity in the event of a mutation. It comigrated with the indicated 234 bp fragment of the marker (M). It can be seen clearly that no tumor DNA contained a major fragment comigrating with the control, while two smaller fragments of identical size were observed in all tumor samples. Faint bands of higher molecular weight became visible by the highly sensitive silver stain. These bands most likely reflect annealing artifacts of the primers, given that the electrophoretic mobility was lower than that of the control. Alternatively, these fragments may represent traces of undigested DNA due to incomplete digestion of wild-type DNA by EcoRV. They do not indicate a heterozygous state (mutant and wildtype) of tumor cells, because $50 \%$ of mutated tumor DNA would have yielded band intensities similar to that observed in lane C.

Abbreviations: GBM, glioblastoma multiforme; Flt3, fms-like tyrosine kinase-3; Flt3L, fms-like tyrosine kinase-3 ligand.

amounts of the ligand during immunostimulation would be expected eventually to saturate all receptors present in the plasma membranes, leading to maximal activation of this pathway. This activation would be restricted only by the amount of receptor protein, but no longer regulated by endogenous concentrations of the ligand. Therefore, an important prerequisite of this novel immunostimulating therapy would be restriction of receptor expression within the tumor tissue to the antigen-presenting dendritic cells, which are the intended targets. 
This prerequisite was not fulfilled ideally. Although in the present study only trace amounts of the receptor at the mRNA level and no activating mutations were observed in human astrocytic tumors from adult patients (World Health Organization grades II-IV), it cannot be excluded at this point that the pathway may play a role in proliferation of glioma cells, the cell cycle, and eventually apoptosis. Expression of the receptor and ligand should be investigated in more detail at the protein level, including quantification by enzymelinked immunosorbent assays. Moreover, the responsiveness of glioma cells in defined fetal calf serum-free medium to Flt3L with respect to cell proliferation, cell cycle, and apoptosis should be measured. To our knowledge, such data are missing in the animal studies.

\section{Acknowledgment}

We gratefully acknowledge the excellent technical assistance of Ines Schellhase.

\section{Disclosure}

The authors report no conflicts of interest in this work.

\section{References}

1. De Witt Hamer PC. Small molecule kinase inhibitors in glioblastoma: a systematic review of clinical studies. Neuro Oncol. 2010;12: 304-316.

2. Ohgaki H, Kleihues P. Genetic pathways to primary and secondary glioblastoma. Am J Pathol. 2007;170:1445-1453.

3. Fan QW, Weiss WA. Targeting the RTK-PI3K-mTOR axis in malignant glioma: overcoming resistance. Curr Top Microbiol Immunol. 2010;347:279-296.

4. Hegi ME, Diserens AC, Gorlia T, et al. MGMT gene silencing and benefit from temozolomide in glioblastoma. $N$ Engl J Med. 2005;352: 997-1003.

5. Hainsworth JD, Shih KC, Shepard GC, Tillinghast GW, Brinker BT, Spigel DR. Phase II study of concurrent radiation therapy, temozolomide, and bevacizumab followed by bevacizumab/everolimus as first-line treatment for patients with glioblastoma. Clin Adv Hematol Oncol. 2012;10:240-246.
6. Hegi ME, Diserens AC, Bady P, et al. Pathway analysis of glioblastoma tissue after preoperative treatment with the EGFR tyrosine kinase inhibitor gefitinib - a phase II trial. Mol Cancer Ther. 2011;10: 1102-1112.

7. Kreisl TN, Smith P, Sul J, et al. Continuous daily sunitinib for recurrent glioblastoma. J Neurooncol. 2013;111:41-48.

8. Reardon DA, Vredenburgh JJ, Coan A, et al. Phase I study of sunitinib and irinotecan for patients with recurrent malignant glioma. J Neurooncol. 2011;105:621-627.

9. Ali S, Curtin JF, Zirger JM, et al. Inflammatory and anti-glioma effects of an adenovirus expressing human soluble Fms-like tyrosine kinase 3 ligand (hsFlt3L): treatment with hsFlt3L inhibits intracranial glioma progression. Mol Ther. 2004;10:1071-1084.

10. Ali S, King GD, Curtin JF, et al. Combined immunostimulation and conditional cytotoxic gene therapy provide long-term survival in a large glioma model. Cancer Res. 2005;65:7194-7204.

11. Ghulam Muhammad AK, Candolfi M, King GD, et al. Antiglioma immunological memory in response to conditional cytotoxic/immunestimulatory gene therapy: humoral and cellular immunity lead to tumor regression. Clin Cancer Res. 2009;15:6113-6127.

12. King GD, Muhammad AK, Curtin JF, et al. Flt3L and TK gene therapy eradicate multifocal glioma in a syngeneic glioblastoma model. Neuro Oncol. 2008;10:19-31.

13. Hurtt MR, Moossy J, Donovan-Peluso M, Locker J. Amplification of epidermal growth factor receptor gene in gliomas: histopathology and prognosis. J Neuropathol Exp Neurol. 1992;51:84-90.

14. Nishikawa R, Ji XD, Harmon RC, et al. A mutant epidermal growth factor receptor common in human glioma confers enhanced tumorigenicity. Proc Natl Acad Sci U S A. 1994;91:7727-7731.

15. Akhavan D, Cloughesy TF, Mischel PS. mTOR signaling in glioblastoma: lessons learned from bench to bedside. Neuro Oncol. 2010;12: 882-889

16. Huang HS, Nagane M, Klingbeil CK, et al. The enhanced tumorigenic activity of a mutant epidermal growth factor receptor common in human cancers is mediated by threshold levels of constitutive tyrosine phosphorylation and unattenuated signaling. J Biol Chem. 1997;272: 2927-2935.

17. Mellinghoff IK, Wang MY, Vivanco I, et al. Molecular determinants of the response of glioblastomas to EGFR kinase inhibitors. $N$ Engl J Med. 2005;353:2012-2024.

18. Gilliland DG, Griffin JD. Role of FLT3 in leukemia. Curr Opin Hematol. 2002;9:274-281.

19. Nakao M, Yokota S, Iwai T, et al. Internal tandem duplication of the flt 3 gene found in acute myeloid leukemia. Leukemia. 1996;10: 1911-1918

20. Weisberg E, Sattler M, Ray A, Griffin JD. Drug resistance in mutant FLT3-positive AML. Oncogene. 2010;29:5120-5134.
OncoTargets and Therapy

\section{Publish your work in this journal}

OncoTargets and Therapy is an international, peer-reviewed, open access journal focusing on the pathological basis of all cancers, potential targets for therapy and treatment protocols employed to improve the management of cancer patients. The journal also focuses on the impact of management programs and new therapeutic agents and protocols on

\section{Dovepress}

patient perspectives such as quality of life, adherence and satisfaction. The manuscript management system is completely online and includes a very quick and fair peer-review system, which is all easy to use. Visit http://www.dovepress.com/testimonials.php to read real quotes from published authors. 